Résumés des conférences et travaux

\title{
Art et archéologie de la Chine pré-imperiale
}

\section{Alain Thote}

\section{OpenEdition \\ Journals}

Édition électronique

URL : https://journals.openedition.org/ashp/3961

DOI : 10.4000/ashp.3961

ISSN : 1969-6310

Éditeur

Publications de l'École Pratique des Hautes Études

\section{Édition imprimée}

Date de publication : 1 septembre 2020

Pagination : 328-333

ISSN : 0766-0677

Référence électronique

Alain Thote, "Art et archéologie de la Chine pré-imperiale ", Annuaire de l'École pratique des hautes études (EPHE), Section des sciences historiques et philologiques [En ligne], 151 | 2020, mis en ligne le 09 juillet 2020, consulté le 06 juillet 2021. URL : http://journals.openedition.org/ashp/3961 ; DOI : https:// doi.org/10.4000/ashp.3961 


\title{
ART ET ARCHÉOLOGIE DE LA CHINE PRÉ-IMPERIALE
}

\author{
Directeur d'études : M. Alain Thоте, \\ membre de l'Institut
}

Programme de l'année 2018-2019: I. Les cultures régionales de la péninsule du Shandong et du Jiangnan aux $X^{e}-I I I^{e}$ s. av. J.-C. (suite). - II. Actualité des recherches archéologiques en Chine.

\section{Les cultures régionales de la péninsule du Shandong et du Jiangnan aux $X^{\mathrm{e}}-I I I^{\mathrm{e}}$ s. $a v . J .-C$.}

Les deux royaumes de $\mathrm{Wu}$ 吳 et de Yue 越 dont les territoires couvraient la région $\mathrm{du}$ cours inférieur du fleuve Bleu (Yangzi) sont connus historiquement à partir du $\mathrm{VI}^{\mathrm{e}}$ s. av. J.-C. Ils ont souvent été plongés dans des guerres, mais tandis que Yue était excentré, et donc moins exposé, Wu s'est beaucoup impliqué dans les affaires des pays de la plaine Centrale et du royaume de $\mathrm{Chu}$, jusqu'à tenter de conquérir ce dernier. Finalement, trop ambitieux sans doute et ayant dispersé ses forces, Wu fut vaincu par Yue en 473 av. J.-C. Puis ce fut au tour de Yue d'être détruit par le royaume de Chu qui l'annexa en 333 av. J.-C. La proximité géographique de Wu et de Yue, les rapports étroits qu'ils entretenaient et leur sinisation progressive rendent difficile une caractérisation culturelle de chacun. Tout au plus est-il manifeste que les pays de culture Zhou ont exercé une influence sur Wu beaucoup plus tôt et de manière plus intense que sur Yue. Comme nous l'avions vu l'année précédente, les textes décrivent de manière allusive les habitants de la région, leurs coutumes, leur mode de vie, et le peu qu'ils en disent fut écrit par des Chinois de la plaine Centrale, influencés par leurs préjugés à l'encontre de populations qu'ils considéraient comme «barbares », sans avoir jamais eu l'occasion de les observer dans leur pays. La culture matérielle nous éclaire davantage sur les populations qu'on appelait les Yue 越, ou Bai Yue 百越, « les Cent Yue », une expression qui souligne la diversité de peuplement et de culture de toute la région située au sud du fleuve Bleu depuis la région de Shanghai jusqu'au Vietnam. À ce sujet, on se reportera au rapport de l'année 2017-2018 publié en 2019 dans l'Annuaire de la section des SHP de l'EPHE, p. 398-404.

La conférence a porté principalement sur les bronzes de Wu et de Yue, avec pour problématiques la recherche des premières productions de leurs ateliers et une identification des styles locaux. Les régions concernées sont le Centre et le Sud du Jiangsu, le Nord du Zhejiang, et le Sud de l'Anhui. Les débuts de la métallurgie du bronze dans ces régions ont été stimulés par des échanges indirects avec la Chine centrale, mais surtout avec la région du cours moyen du fleuve Bleu. Dès le milieu du deuxième millénaire, la quête de nouvelles ressources en cuivre et en étain avaient en effet conduit les Shang (env. 1550-1050 av. J.-C.) à établir des contacts étroits avec cette dernière (provinces actuelles du Hubei et du Hunan, site de Panlongcheng 盤龍城 près de 
Wuhan), et à pénétrer jusque dans la partie nord du Jiangxi, où s'est alors développée une métallurgie de très haute qualité. De proche en proche, le long du fleuve, les réalisations de ce centre, puis la technique même ont gagné la région du Bas Yangzi. Plusieurs découvertes dans le Jiangsu et le nord du Zhejiang témoignent de cette diffusion. Ce sont notamment, pour les plus anciens bronzes, des cloches de type nao 鐃 et des vases du XIII ${ }^{\mathrm{e}}$ s. av. J.-C., caractéristiques des ateliers du Nord du Hunan.

La région nord du Jiangxi a certainement joué un rôle important dans la diffusion de la métallurgie vers la région du Bas Yangzi. Le mobilier de la grande tombe de Dayangzhou, district de Xin'gan, 新幹大洋洲 dans le Nord du Jiangxi témoigne tout particulièrement de la qualité exceptionnelle de la fonte locale au XIII ${ }^{\mathrm{e}} \mathrm{s}$. av. J.-C.

Les quarante-huit bronzes rituels de cette tombe, fondus selon les techniques inventées en Chine centrale, ont plusieurs traits distinctifs, qui ont été analysés. D'après les parallèles tracés avec les bronzes de la plaine Centrale, pour autant qu'on admette qu'ils avaient le même usage, ce sont trente-huit vases pour cuire les offrandes de nourriture (30 ding 鼎, dont 6 fangding 方鼎, 2 ding en forme de pou 瓿; 5 li 鬲; 3 yan 甗), 1 dou 豆 pour consommer des mets, huit vases pour stocker la boisson (2 you 卢, 1 fangyou 方卢, $2 \mathrm{hu}$ 壼), à recueillir l'eau des ablutions (1 pan 盤). Ces proportions s'avèrent très différentes de celles qu'on observe dans les ensembles rituels d'Anyang dont ils sont à peu près contemporains. À Dayangzhou, la typologie est dominée par des tripodes ding et $l i$ pour la cuisson des offrandes de nourriture, soit plus de $73 \%$ des vases. Aucun ding ne suit le même modèle, et tous sont de dimensions différentes. Les vases pour la boisson sont complètement absents. Autre caractère distinctif, les vases en bronze étaient complétés par une vaisselle en grès à couverte. À la même époque, à Anyang, les ensembles rituels contiennent en majorité des vases pour la boisson, les ding n'en constituent qu'une faible partie. D'autre part, les fangding et les ding à pieds laminaires représentent deux tiers des vases de Dayangzhou quand ces formes sont minoritaires à Anyang. On pourrait en déduire que l'ensemble de bronzes de Dayangzhou est constitué de pièces dépareillées, réunies au hasard grâce à des prises de butin par exemple. Il n'en est rien, car leur style très homogène est différent du style des bronzes contemporains. Le répertoire des motifs zoomorphes se distingue aussi de celui des artistes Shang, réservant une moindre place au taotie 饕䬸. Le décor des bronzes comprend des sculptures d'animaux en ronde bosse, rendus de manière naturaliste dans un style opposé à l'esprit des bronzes Shang. Les motifs secondaires ajoutent une note particulière à ces traits distinctifs, notamment les chevrons et les spirales en relief.

Le style des bronzes de Dayangzhou présente un caractère homogène, leur qualité est sans exception de très haut niveau, et leur fonte originale. Or parmi les milliers de bronzes découverts à Anyang, quelques-uns entretiennent d'incontestables affinités de style avec ceux de Dayangzhou. Le fait qu'ils portent une inscription suggère l'idée qu'ils ont certainement été fondus en Chine centrale, bien que leur style laisse plutôt supposer une provenance méridionale. Pour lever cette contradiction, on peut émettre l'hypothèse que des artisans qualifiés sont montés de la région nord du Jiangxi jusqu'à Anyang en y apportant leur savoir-faire et leur style. Dans ces conditions, des transferts artistiques se seraient produits depuis la région du cours moyen du fleuve Bleu en territoire Shang. Une recherche plus approfondie mériterait d'être 
entreprise sur ce sujet, comme du reste des chercheurs l'ont déjà suggéré. La question reste de savoir pourquoi certains éléments plutôt que d'autres pénétraient ainsi en Chine centrale ${ }^{1}$.

L'une des caractéristiques les plus originales des vases en bronze de Dayangzhou apparaît dans les arêtes découpées soulignant leur profil. Par leur découpe et leurs volutes, celles-ci contribuent beaucoup à l'enrichissement du décor. À Anyang, les arêtes restent au contraire très sobres. Or, à la toute fin des Shang, un style que Jessica Rawson a qualifié de " flamboyant » est apparu soudainement en Chine centrale et dans la vallée de la Wei (région de Baoji 寶雞, par exemple). Dans ce style, on décèle des emprunts aux productions du centre métallurgique d'où les bronzes de Dayangzhou étaient issus, bien que deux siècles les en séparent. Il est probable que ces ateliers, actifs aux XIII ${ }^{\mathrm{e}}-\mathrm{XI}^{\mathrm{e}}$ siècles, furent à l'origine de la diffusion de ce style lors des grands bouleversements consécutifs à la conquête du pouvoir par les Zhou sur les Shang. On a observé que durant cette courte période de nouvelles voies de commerce se sont ouvertes entre la Chine méridionale et la Chine centrale. Les échanges sont bien attestés par la présence en abondance de grès à couverte, originaires de la région du bas Yangzi, notamment des coupes dou faites toujours sur le même modèle, dans les tombes de l'aristocratie Zhou, aussi bien dans les cimetières du nord du Hubei et du Henan qu'à Zhouyuan 周源 (Shaanxi).

Afin de tester cette hypothèse, nous avons passé en revue les bronzes de plusieurs sites de la fin des Shang. Cela nous a permis de confirmer ces liens, tout en suivant l'une de ces voies d'échanges passant dans la vallée de la Han, qui faisait une jonction naturelle entre la partie occidentale du Hubei et le sud du Shaanxi, pour aboutir à Baoji. Ces échanges ne se sont pas limités aux objets mêmes, ils ont certainement impliqué des métallurgistes amenés à quitter leur région d'origine pour exercer leurs talents dans la région de Zhouyuan et sa périphérie au début de la dynastie Zhou.

Le plus ancien bronze Zhou découvert dans la région de $\mathrm{Wu} /$ Yue n'est autre que le Yi Hou Ze gui 宜侯失笽 (H. 15,7 cm, diam. 22,5 cm), découvert à Yandunshan, district de Dantu 丹徒煙墩山, en 1954, près de Zhenjiang sur la rive droite du fleuve $\mathrm{Bleu}^{2}$. Il se trouvait dans une cache (ou une tombe mal identifiée) contenant plusieurs bronzes datables entre environ le $\mathrm{XI}^{\mathrm{e}} \mathrm{s}$. et le $\mathrm{VIII}^{\mathrm{e}} \mathrm{s}$. av. J.-C. Ce vase porte une longue inscription (118 à 126 caractères, selon les auteurs) qui relate une cérémonie d'investiture révélant comment les premiers rois Zhou parvenaient à contrôler leur territoire par le biais de fiefs attribués aux hommes qui avaient fait leurs preuves et leur étaient fidèles ${ }^{3}$. L'inscription, que nous avons traduite avec les étudiants, relate le transfert d'un prince appelé Ze 失 de son territoire d'origine, le domaine de Yu 虞, vers un nouveau territoire, le domaine de Yi 宜. La cérémonie se tient dans le temple ancestral de

1. Une étude récente sur le fangding de Dayangzhou dont la partie inférieure forme un brasero montre qu'à partir de cette création par les artisans métallurgistes de la région nord du Jiangxi, plusieurs bronzes similaires ou proches ont été introduits en Chine centrale, et ont inspiré à leur tour la fonte de bronzes reprenant l'association récipient / brasero. Cf. Lang Jianfeng 郎劍鋒, «Qingtong “zao-ding” yanjiu » (青銅 “燥鼎” 研究), Kaogu, 10 (2018), p. 85-100.

2. Voir Wenwu ziliao congkan, 1955.5, Kaogu xuebao, 9 (1955). Ces publications ont été suivies par une quantité d'autres articles, ce bronze ayant fait couler beaucoup d'encre.

3. Edward L. Shaughnessy, " Historical Geography and the Extent of the Earliest Chinese Kingdoms », Asia Major, vol. 2, 2 (1989), p. 1-22. Voir p. 13. 
Yi (soit à Yi même, soit à Zhouyuan dans la vallée de la Wei), sous l'autorité du roi, très certainement Kang 周康王 qui régna de 1005 à 978 av. notre ère. D'après son style, ce vase a été fondu dans la région de Zhouyuan, mais pas localement.

Les autres bronzes rituels de Yandunshan ont été analysés. Plusieurs présentent des traits originaux, témoins d'une facture provinciale, mais sont de qualité moyenne, voire médiocre. Ils se distinguent bien d'un vase comme le Yi Hou Ze gui. Dans cet ensemble, une verseuse gong 觥 semble aussi représenter une exception en raison de son beau modelé en forme d'animal et de son excellente fonte. Nous avons montré qu'il venait aussi d'un atelier de la vallée de la Wei, mais que son couvercle avait été fondu localement pour remplacer le couvercle d'origine. Ces bronzes originaires de la vallée de la Wei ont servi de modèles aux artisans de Wu. En effet, un autre vase gong découvert avec le précédent s'est révélé être une pâle copie locale de ce dernier. Ces comparaisons, et d'autres encore, montrent que jusqu'au viII ${ }^{\mathrm{e}}$ s. av. J.-C. au moins, le pays de Wu n'avait pas encore développé une fonte de qualité : les métallurgistes se contentaient de copier, avec des moyens insuffisants, les modèles que le hasard des échanges leur apportait sous les yeux, souvent des bronzes plus anciens venant d'ateliers métropolitains. Ce n'est qu'à partir du $\mathrm{vI}^{\mathrm{e}} \mathrm{s}$. av. J.-C. que la métallurgie de $\mathrm{Wu}$ prit son essor, comparable en qualité à celle de Chu à laquelle elle fit de nombreux emprunts, tout en développant un style propre.

Nous nous sommes ensuite interrogés à propos des centres métallurgiques de la région sud de l'Anhui, d'abord sur la rive nord du Yangzi, puis sur la rive sud. La carte des sites révèle une concentration particulière de sites sur les deux rives, l'arrière-pays étant constitué de zones de montagnes très denses. Sans doute la proximité du Yangzi favorisait-elle les échanges locaux et les communications à longue distance avec les établissements de culture plus riche situés en amont. Le style des bronzes de cette micro-région aux $\mathrm{VIII}^{\mathrm{e}}-\mathrm{VII}^{\mathrm{e}}$ S. av. J.-C. est immédiatement reconnaissable : les motifs de dragons s'inspirent des bronzes de la plaine centrale et de la vallée de la Wei, mais transformés. Leur dessin manque de nervosité et apparaît schématique au premier regard. Cependant, on ne peut juger ces bronzes selon les critères de la métropole, car ils ont dans leur singularité des qualités propres. Des préoccupations d'ordre esthétique y sont bien présentes, par exemple dans le profil des vases souligné par des arêtes découpées, un trait stylistique hérité de la région nord du Jiangxi. De plus, ces ateliers ont inventé des formes qui se sont largement diffusées par la suite. Parmi celles-ci, le tripode à bec verseur pourvu d'une anse très large, jiaohe 鐎盉, a connu une belle fortune dans toute la région et plus largement dans le royaume de Chu. Le bec prend la forme d'une tête d'animal tandis que l'anse en forme le corps. La typologie des bronzes de cette région reste cependant limitée en comparaison à celle des cultures de Chu et de la plaine Centrale.

En 1959, un cimetière a été découvert près de la ville de Tunxi 屯溪, tout au sud de l'Anhui, dans une région très isolée. Les tombes étaient creusées chacune dans un tumulus construit par l'homme. Ce sont les plus anciennes tombes de Chine sur ce modèle. Entre 1959 et 1975 huit grandes tombes ont été fouillées, auxquelles s'ajoutent de petites tombes ${ }^{4}$. Leur mode de construction, typique de Yue, reste unique

4. Li Guoliang 李國梁 (éd.), Tunxi tudunmu fajue baogao (屯溪土墩墓發掘報告), Hefei, Anhui renmin chubanshe, 2006. 
en Chine à l'âge du Bronze. À côté de deux vases you provenant de la vallée de la Wei, tous les autres vases sont de facture locale. Aucun ne ressemble de près ou de loin aux bronzes découverts le long des deux rives du Yangzi. Ce sont des bronzes typique du pays de Yue. Certains copient les you de la vallée de la Wei, mais en leur donnant une très forte coloration locale, d'autres reproduisent des vases zun 尊 selon les canons Shang, alors qu'en Chine centrale cette forme avait complètement disparu dès le $\mathrm{IX}^{\mathrm{e}} \mathrm{s}$. av. J.-C. Le décor en surface de ces bronzes est foisonnant, d'une finesse inconnue ailleurs en Chine, au point de privilégier l'effet sur la lisibilité. On ne distingue plus de motifs zoomorphes dans le relief des registres, qui reproduit plutôt l'entrelacement des éclisses de vannerie. En bref, ces motifs, ces décors n'appartiennent qu'aux ateliers de Yue. Certaines formes privilégiées dans cette culture, comme les you, les zun, les gui 筬, sont des survivances du passé. D'autres vases, d'aspect classique, sont cependant infidèles à leur modèle, car ils ne servent plus la même fonction dans leur culture d'adoption. C'est le cas de « bassins » pan qui ont une paroi ajourée et qui de ce fait ne peuvent pas recueillir l'eau des ablutions. Des coupes $y u$ 孟 en bronze sont des imitations de grès à couverte à panse carénée. Mais ce ne sont pas seulement les ensembles rituels qui, dans leur typologie, leur décor, le nombre de pièces de chaque type, signalent l'originalité de la culture des élites de Yue, mais aussi l'armement. Les tombes de Tunxi ne comprenaient quasiment aucune hache-poignard, mais des épées courtes (env. 37-46 cm de long), sur le modèle qui allait s'imposer au v $\mathrm{v}^{\mathrm{e}} \mathrm{s}$. av. J.-C. dans toute la Chine.

Les deux dernières séances de l'année ont été consacrées à l'analyse de l'architecture des tombes à tumulus de Yue.

\section{Actualité des recherches archéologiques en Chine}

\section{Le site de Shimao, district de Shenmu, municipalité de Yulin 榆林市神木縣石峁 (Shanxi)}

Identifié en 1958, ce site focalise toute l'attention des chercheurs aujourd'hui, car les résultats des fouilles qui y ont été récemment menées bouleversent complètement les idées qu'on se faisait sur la transition en Chine entre le néolithique et l'âge du Bronze. Il s'agit d'une cité, en activité entre 2300 et 1800 av. J.-C., construite partiellement en pierre : fortifications, terrasses y ont été découvertes enfouies sous la terre qui les a préservées à travers les siècles. Or, la pierre n'était jamais utilisée dans la plaine Centrale, aussi bien pour la construction de remparts que pour celle d'architectures monumentales. On lui a toujours préféré la terre damée, mais ce choix était en partie imposé par les conditions environnementales.

Ces dernières années, les archéologues ont découvert dans la cité palatiale de Shimao un atelier de fabrication d'objets en os. Celui-ci était spécialisé dans une production de masse, ce qui signifie qu'une forme de commerce avait pris place sous le contrôle des élites locales dès la fin du Néolithique. La présence de certaines sculptures sur pierre incorporées dans la paroi des terrasses ou dans celle des palais ne manque pas non plus d'intriguer les archéologues. Elles sont toutes indépendantes les unes des autres et placées dans le mur sans qu'un ordre soit immédiatement perceptible. Il s'agit en particulier d'une paire d'yeux découverte en 2015, d'un serpent, 
d'un grand masque zoomorphe vu de face encadré par deux têtes de même nature vues de profil. Cette dernière composition rappelle le décor des bronzes d'Erligang 二里崗 (municipalité de Zhengzhou 鄭州, Henan). Or la civilisation d'Erligang n'est apparue que vers 1500 av. J.-C., soit trois siècles après l'abandon du site de Shimao. Il est clair qu'un lien, indirect en raison du décalage chronologique, a existé entre Shimao et la région de Zhengzhou. Enfin, à côté de ces associations stylistiques, un autre fait mérite d'être signalé, la présence de crânes humains dans une fosse, attestant de sacrifices selon un mode qui évoque immédiatement les sacrifices Shang à Anyang (env. 1300-1050 av. J.-C.) dans l'enceinte des grandes tombes royales ou sous les palais / temples de la cité. Pourtant, de telles pratiques restent peu attestées durant les siècles intermédiaires tant à Erlitou 二里頭 qu'à Erligang.

Plusieurs facteurs permettent de déterminer que le site est représentatif d'une société complexe. Ses remparts défensifs, les portes monumentales qui y sont percées, la conception du plan de la cité comprenant une enceinte pour le palais, suggèrent une organisation urbaine sophistiquée. Ces constructions d'envergure ont impliqué la mobilisation d'ouvriers en très grand nombre. Les sacrifices humains témoignent d'un recours à la violence qui était jusqu'à présent sous-estimé pour cette époque de transition. La spécialisation de l'artisanat engagé dans une production de masse est l'indice d'une division poussée du travail. Aucun de ces points ne concorde vraiment avec l'idée qu'on se faisait jusqu'à présent des premières cités qui fleurirent en différentes régions de la Chine à la fin du néolithique.

\section{Les cultures de la Chine du Nord-Est}

Durant six semaines, le directeur d'études a effectué une mission dans le Nord-Est de la Chine, invité par l'université du Jilin. Ce séjour lui a permis de visiter de nombreux sites, des musées, des centres archéologiques, et de découvrir des cultures souvent encore mal publiées. Une présentation en a été faite au cours de deux séances. 